\title{
Management of cerebral hemorrhage resulting from the side effect of mRNA vaccines
}

\author{
A short perspective
}

Pardis Tabaee Damavandi

\begin{abstract}
Author Note
Copyright: $(\odot$ the author(s), publisher and licensee. This is an open-access article distributed under the terms of the Creative Commons Attribution Non-Commercial License, which permits unrestricted non-commercial use, distribution, and reproduction in any medium, provided the original work is properly cited
\end{abstract}

\section{Introduction}

Messenger Ribonucleic Acid (mRNA) vaccines ${ }^{[1]}$ have latterly demonstrated progressive notoriety due to the modern coronavirus (Covid-19); they were also made popular in the $1990 \mathrm{~s}^{[2]}$ possibly due to advances in gene therapy.

Although the microbiology, pharmacy and pharmacology community remains cynical towards the novel technology, whereby the live attenuated vaccine temperings are preferred, due to their improved pharmacovigilance profiles, mRNA vaccination has already been produced and been administered worldwide.

Traditional methods are generally safer to date and therefore still the primary choice for vaccine development.

Nevertheless, important side effects have been reported of thrombocytopenia ${ }^{[3]}$ and cerebral venous thrombosis hemorrhage linked to the depletion of platelets required to maintain vessel strength and which factor in coagulation. Following, a potential rationale why this side effect is observed and why the traditional methods would be safer and possible management of adverse events are further discussed.

\section{Rationale}

The rationale behind why "radical" side effects are often reported in which there is cellular depletion at the level of the bone marrow, whether apoptotic or necrotic, posterior to mRNA vaccine inoculation could be a result of a simple intercalation. This hypothesis is not only analogical, to chemotherapeutic agents ${ }^{[4]}$ intercalating nucleic acids inducing apoptosis and which also show adverse effects on suppressing the production of corpuscular elements of the blood by the bone marrow, but also structural-functional. A vaccine is purposefully created for recognition by the organism and to suscitate immunity and remember it; this process is aimed at ultimately enhancing phagocytosis. The failure to achieve this outcome by the inoculum, may lead to anaphylaxis, however, in the case of a mRNA vaccine we could think that the bone marrow which is constantly generating blood elements, with a frequent turnover, may subtract the mRNA from the vaccine and use it for that purpose. This "misuse" 
is not going to succeed at that level and could lead to necrosis or apoptosis and thus thrombocytopenia since the mRNA is foreign to the host organism.

\section{Complications of thrombocytopenia}

The most challenging complication of thrombocytopenia observed in the novel coronavirus vaccine is associated with cerebral vein thrombosis, which is difficult to manage although it can be attempted; platelet loss can be also managed with splenectomies or corticosteroid use but again remains "uneasy". Bleeding is the major "symptom" observed. These side effects are not normally found in the live attenuated virus vaccines, although neurovirulence is instead reported in conventional vaccines which is often thought to be an exacerbation of the disease itself ${ }^{[5]}$. Cerebral venous thrombosis has likewise been detected in herpes zoster virus $^{[6]}$.

\section{Potential Management of Cerebral Vein Thrombosis}

Although no studies suggest that this may be effective, Cerebrolysin (developed by an Austrian scientist in the late 40s) and Apomorphine, which are commonly used to treat traumatic brain injury $(\mathrm{TBI})^{[7]}$ and coma $^{[8]}$ could be employed to counteract cerebral hemorrhage. Manipulation of catecholamine transmission, osmotic compounds, vitamins, could be an alternative strategy but with risks, as well as plasmapheresis. Nevertheless the conventional vaccines, and more specifically the live attenuated ones, remain a favoured option compared to the novel ones. But, mRNA technology could be adjusted within applications in bone marrow dysfunctions perhaps as additional stabilisers in chemotherapy treatment.

\section{Conclusion}

mRNA vaccine administration has important side effects including thrombocytopenia, which have been observed in other types of vaccines (e.g

recombinant), and affects primarily the CNS. This seems to lead to cerebral vein thrombosis which is harder to manage compared to the side effects caused by traditional live attenuated vaccines. Cerebrolysin, Apomorphine, commonly used for TBI and comatose patients, vitamins too (Vitamin K, D) could be used to obviate that problem however no studies have been carried out to date.

No conflicts of interest to disclose

\section{References}

1. World Health Organization. (2020). mRNA vaccines against COVID-19: Pfizer-BioNTech COVID-19 vaccine BNT162b2: prepared by the Strategic Advisory Group of Experts (SAGE) on immunization working group on COVID-19 vaccines, 22 December 2020. World Health Organization. https://apps.who.int/iris/handle/10665/338096 
2. Wolff, J. A., Malone R. W., Williams P. et al. Direct gene transfer into mouse muscle in vivo. Science 247, 1465-1468 (1990). This study demonstrates protein production from RNA administered in vivo.

3. Bhattacharjee $S$, Banerjee $M$. Immune Thrombocytopenia Secondary to COVID-19: a Systematic Review. SN Compr Clin Med. 2020 Sep 19:1-11. doi: 10.1007/s42399-020-00521-8. Epub ahead of print. PMID: 32984764; PMCID: PMC7501509.

4. Weycker, D., Hatfield, M., Grossman, A. et al. Risk and consequences of chemotherapy-induced thrombocytopenia in US clinical practice. BMC Cancer 19, 151 (2019). https://doi.org/10.1186/s12885-019-5354-5

5. Horien C, Grose C. Neurovirulence of varicella and the live attenuated varicella vaccine virus. Semin Pediatr Neurol. 2012 Sep;19(3):124-9. doi: 10.1016/j.spen.2012.02.006. PMID: 22889542; PMCID: PMC3419367.

6. Gilden DH, Mahalingam R, Cohrs RJ, Kleinschmidt-DeMasters BK, Forghani B. The protean manifestations of varicella-zoster virus vasculopathy. J Neurovirol. 2002 Dec;8 Suppl 2:75-9. doi: 10.1080/13550280290167902. PMID: 12491155.

7. Brainin M. Cerebrolysin: a multi-target drug for recovery after stroke. Expert Rev Neurother. 2018 Aug;18(8):681-687. doi: 10.1080/14737175.2018.1500459. Epub 2018 Jul 18. PMID: 30004268.

8. Fridman EA, Krimchansky BZ, Bonetto M, Galperin T, Gamzu ER, Leiguarda $\mathrm{RC}$, Zafonte R. Continuous subcutaneous apomorphine for severe disorders of consciousness after traumatic brain injury. Brain Inj. 2010;24(4):636-41. doi: 10.3109/02699051003610433. PMID: 20235766. 